\title{
ON SUPERSOLVABLE GROUPS WHOSE MAXIMAL SUBGROUPS OF THE SYLOW SUBGROUPS ARE SUBNORMAL
}

\author{
PENGFEI GUO, XINGQIANG XIU, AND GUANGJUN XU
}

\begin{abstract}
A finite group $G$ is called an $\mathrm{MSN}^{*}$-group if it is supersolvable, and all maximal subgroups of the Sylow subgroups of $G$ are subnormal in $G$. A group $G$ is called a minimal non-MSN*-group if every proper subgroup of $G$ is an $\mathrm{MSN}^{*}$-group but $G$ itself is not. In this paper, we obtain a complete classification of minimal non-MSN* ${ }^{*}$-groups.
\end{abstract}

\section{INTRODUCTION}

Only finite groups are considered in this paper and our notation is standard.

Let $\mathfrak{F}$ be a class of groups. A group $G$ is called a minimal non- $\mathfrak{F}$-group or $\mathfrak{F}$-critical group if all subgroups but $G$ itself belong to $\mathfrak{F}$. The characterization of minimal non- $\mathfrak{F}$-groups plays a critical role in analyzing the structure of groups with certain group theory property. It is important to obtain a detailed knowledge of minimal non-F-groups so that some deep insights into what makes a group belong to $\mathfrak{F}$ may turn to be achievable. Moreover, when proving that a group belongs to $\mathfrak{F}$, researchers can benefit from such descriptions of the minimal non$\mathfrak{F}$-groups by induction or minimal counterexample. Many meaningful results on this topic have been obtained, and they have indeed pushed forward the development of group theory. For example, Schmidt [10] determined the structure of minimal non-nilpotent groups, and Doerk [3] determined the structure of minimal non-supersolvable groups. Ballester-Bolinches and Esteban-Romero [1 provided a complete classification of minimal non-supersolvable groups, which is shown to be useful and hence is exploited in solving the problem studied in this paper.

Srinivasan [12] studied groups in which all maximal subgroups of the Sylow subgroups are normal, and proved that such groups are supersolvable. Later, Walls

2010 Mathematics Subject Classification. 20D10; $20 \mathrm{E} 34$.

Key words and phrases. Subnormal group; Maximal subgroup; MSN*-group; Sylow subgroup; Automorphism.

The first and second authors were supported by the National Natural Science Foundation of China (No. 11661031); the third author was supported by the National Natural Science Foundation of China (No. 11661084) and the Program for Sci-Tec Innovative Talents of Guizhou Province (No. [2015]502). 
[13] called such groups MNP-groups and investigated the structure of the MNPgroups. Recently, Guo et al. [4] determined the complete classification of minimal non-MNP-groups (those groups which are not MNP-groups but whose proper subgroups are all MNP-groups).

On the other hand, Srinivasan 12 also proved that a group $G$ is solvable but not necessarily supersolvable if all maximal subgroups of the Sylow subgroups are subnormal in $G$. The alternating group $A_{4}$ is such an example. Guo et al. [5] called such groups MSN-groups and gave a characterization of minimal non-MSN-groups (defined similarly as minimal non-MNP-groups above). Unfortunately, a complete classification of such groups is still unknown.

Naturally, we consider imposing some weaker conditions on MSN-groups. It is well known that CLT-groups (a group is said to be CLT if it possesses subgroups of every possible order, i.e., it satisfies the converse of Lagrange's theorem) are solvable (see [8]) and that supersolvable groups must be CLT (see [7]). Therefore, we investigate MSN-groups with the CLT-property as well as supersolvable MSNgroups. Specific definitions are as follows.

Definition 1.1. A CLT-group $G$ is called a CMSN-group if all maximal subgroups of the Sylow subgroups of $G$ are subnormal in $G$.

Definition 1.2. A supersolvable group $G$ is called an $\mathrm{MSN}^{*}$-group if all maximal subgroups of the Sylow subgroups of $G$ are subnormal in $G$.

It is clear that $\mathrm{MSN}^{*}$-groups must be CMSN-groups, but CMSN-groups need not to be $\mathrm{MSN}^{*}$-groups. For instance, $G=A_{4} \times C_{2}$ is a non-supersolvable CMSNgroup, where $A_{4}$ is the alternating group and $C_{2}$ is cyclic of order 2 . It is interesting that they are equivalent under the assumption on minimal non- $\mathfrak{F}$-groups although these two subgroups are different.

A group $G$ is said to be a minimal non- $M S N^{*}$-group (respectively, a minimal non-CMSN-group) if every proper subgroup of $G$ is an $\mathrm{MSN}^{*}$-group (respectively, a CMSN-group) but $G$ itself is not. In this paper, the minimal non-MSN*-groups (i.e., minimal non-CMSN-groups) are classified completely.

\section{Preliminary Results}

We collect some definitions and lemmas which will be used in the sequel.

Definition 2.1 ([6, Definition 1.4]). Let $\alpha$ be an automorphism of a group $G$. Then $\alpha$ is a semi-power automorphism of $G$ if there exist elements $a_{1}, a_{2}, \ldots, a_{n}$ which generate $G$ such that $\alpha$ maps $a_{i}$ into a power of $a_{i}$ for all $i \in\{1,2, \ldots, n\}$.

By a result of [15, Theorem 2.7] and its Remark, the following lemma is true.

Lemma 2.1. A group $G$ is an $M S N$-group if and only if $G=H \rtimes K$, where $H$ is a nilpotent normal Hall subgroup of $G, K$ is a group whose Sylow subgroups are cyclic and the maximal subgroups of its Sylow subgroups are normal in $G$. $2.2]$.

Based on Lemma 2.1. the following result follows easily by applying 6, Lemma 
Lemma 2.2. A group $G$ is an $M S N^{*}$-group if and only if $G=H \rtimes K$, where $H$ is a nilpotent normal Hall subgroup of $G, K$ is a group whose Sylow subgroups are cyclic and the maximal subgroups of its Sylow subgroups are normal in $G$, and every element of $K$ induces a semi-power automorphism of order dividing a prime in $H / \Phi(H)$.

Lemma 2.3. Let $G$ be a supersolvable minimal non-MSN*-group. Then $|\pi(G)|$ is 2 , where $\pi(G)$ is the set of all primes dividing the order of $G$.

Proof. Let $\left\{P_{1}, P_{2}, \ldots, P_{s}\right\}$ be a Sylow system of $G$. By the hypothesis, there exists some $P_{i}(1 \leqslant i \leqslant s)$ and a maximal subgroup $P^{*}$ of $P_{i}$ such that $P^{*}$ is not subnormal in $G$. Assume $s \geqslant 3$. If $P_{i}$ is non-cyclic, then $P_{i} P_{j}(j \neq i)$ are MSN*groups. By Lemma 2.2. $P_{i}$ is normal in $P_{i} P_{j}$ and also normal in $G$. Thus, $P^{*}$ is subnormal in $G$, a contradiction. Hence $P_{i}$ is cyclic. In this case, by Lemma 2.2 again, $P^{*}$ is normal in $P_{i} P_{j}(j \neq i)$, and so $P^{*}$ is normal in $G$, a contradiction. Thus, $|\pi(G)|=2$.

Lemma 2.4 ([9, 13.4.3]). Let $\alpha$ be a power automorphism of an abelian group $A$. If $A$ is a p-group of finite exponent, then there is a positive integer $l$ such that $a^{\alpha}=a^{l}$ for all $a$ in $A$. If $\alpha$ is nontrivial and has order prime to $p$, then $\alpha$ is fixed-point-free.

Lemma 2.5 ([5, Lemma 2.9]). If a q-group $G$ of order $q^{n+1}$ has a unique non-cyclic maximal subgroup, then $G$ is isomorphic to one of the following groups:

(I) $C_{q^{n}} \times C_{q}=\left\langle a, b \mid a^{q^{n}}=b^{q}=1,[a, b]=1\right\rangle$, where $n \geqslant 2$;

(II) $M_{q^{n+1}}=\left\langle a, b \mid a^{q^{n}}=b^{q}=1, b^{-1} a b=a^{1+q^{n-1}}\right\rangle$, where $n \geqslant 2$ and $n \geqslant 3$ if $q=2$.

Lemma 2.6 (14, Chapter 3, Theorem 1.1]). A group $G$ is supersolvable if and only if all subgroups of $G$ are CLT-groups.

\section{MAin Results}

In this section, we give the complete classification of minimal non-MSN*-groups.

Theorem 3.1. The minimal non-MSN*-groups are exactly the groups of the following types:

(I) $G=\left\langle x, y \mid x^{p}=y^{q^{n}}=1, y^{-1} x y=x^{r}\right\rangle$, where $r^{q} \not \equiv 1(\bmod p), r^{q^{2}} \equiv$ $1(\bmod p), q \mid p-1, n \geqslant 2$ with $0<r<p$.

(II) $G=\left\langle x, y \mid x^{p q}=y^{q}=1, y^{-1} x y=x^{r}\right\rangle$, where $p \equiv 1(\bmod q), r \equiv 1(\bmod q)$, $r^{q} \equiv 1(\bmod p)$ with $1<r<p$.

(III) $G=\left\langle x, y \mid x^{4 p}=1, y^{2}=x^{2 p}, y^{-1} x y=x^{-1}\right\rangle$ with $p>2$.

(IV) $G=\left\langle x, y, z \mid x^{p}=y^{q^{n-1}}=z^{q}=1, y^{-1} x y=x^{r},[x, z]=1,[y, z]=1\right\rangle$, where $n \geqslant 3, p \equiv 1(\bmod q), r \not \equiv 1(\bmod p), r^{q} \equiv 1(\bmod p)$ with $1<r<p$.

(V) $G=\langle x, y, z| x^{p}=y^{q^{n-1}}=z^{q}=1, y^{-1} x y=x^{r},[x, z]=1, z^{-1} y z=$ $\left.y^{1+q^{n-2}}\right\rangle$, where $n \geqslant 3$ and $n \geqslant 4$ if $q=2, p \equiv 1(\bmod q), r \not \equiv 1(\bmod p)$, $r^{q} \equiv 1(\bmod p)$ with $1<r<p$. 
(VI) $G=P \rtimes Q$, where $Q=\langle y\rangle$ is cyclic of order $q^{n}>1$, with $q \nmid p-1$, and $P$ is an irreducible $Q$-module over the field of $p$ elements with kernel $\left\langle y^{q}\right\rangle$ in $Q$.

(VII) $G=P \rtimes Q$, where $P$ is a non-abelian special p-group of rank $2 m$, the order of $p$ modulo $q$ being $2 m, Q=\langle y\rangle$ is cyclic of order $q^{n}>1, y$ induces an automorphism in $P$ such that $P / \Phi(P)$ is a faithful and irreducible $Q$-module, and $y$ centralizes $\Phi(P)$. Furthermore, $|P / \Phi(P)|=p^{2 m}$ and $\left|P^{\prime}\right| \leqslant p^{m}$.

(VIII) $G=P \rtimes Q$, where $P=\left\langle a_{0}, a_{1}, \ldots, a_{q-1}\right\rangle$ is an elementary abelian $p$-group of order $p^{q}, Q=\langle y\rangle$ is cyclic of order $q^{n}, q$ is the highest power of $q$ dividing $p-1$ and $n>1$. Define $a_{j}^{y}=a_{j+1}$ for $0 \leqslant j<q-1$ and $a_{q-1}^{y}=a_{0}^{i}$, where $i$ is a primitive $q$-th root of unity modulo $p$.

Proof. Assume that $G$ is a minimal non-MSN*-group. Since every proper subgroup of $G$ is an MSN*-group, $G$ is supersolvable or minimal non-supersolvable by the definition. By applying a result of [1. Theorems 9, 10] and Lemma $2.3,|\pi(G)|$ is 2 or 3 , and $G$ has a unique normal Sylow subgroup.

We first consider the case of $G$ with $|\pi(G)|=2$, and assume $G=P Q$ with $P \unlhd G$ and $Q \not G$, where $P \in \operatorname{Syl}_{p}(G)$, and $Q \in \operatorname{Syl}_{q}(G)$. Since all the Sylow $q$-subgroups are conjugate in $G$, we only consider the case that $Q$ acts on $P$.

There are four situations, as follows.

(1) Assume that $P=\langle x\rangle$ and $Q=\langle y\rangle$, with $|x|=p^{m},|y|=q^{n}$, and $p>q$.

In this case, $G$ is metacyclic, $y^{-1} x y=x^{r}$ with $r^{q^{n}} \equiv 1\left(\bmod p^{m}\right), q \mid p-1$, $0<r<p^{m}$, and $\left(p^{m}, q^{n}(r-1)\right)=1$. Since $\left\langle y^{q}\right\rangle$ is not subnormal in $G$, it follows that $\left(y^{q}\right)^{-1} x y^{q}=x^{r^{q}} \neq x$. So $r^{q} \not \equiv 1\left(\bmod p^{m}\right)$. The subnormality of $\left\langle y^{q^{2}}\right\rangle$ in $\langle x\rangle\left\langle y^{q}\right\rangle$ implies that $\left\langle y^{q^{2}}\right\rangle$ is normal in $\langle x\rangle\left\langle y^{q}\right\rangle$ by Lemma 2.2 So $\left(y^{q^{2}}\right)^{-1} x y^{q^{2}}=$ $x^{r^{q^{2}}}=x$. Hence $r^{q^{2}} \equiv 1\left(\bmod p^{m}\right)$ and $y$ induces a power automorphism of order $q^{2}$ on $P$. Surely, $y^{q}$ induces a power automorphism of order $q$ on $P$ and every proper subgroup of $\left\langle y^{q}\right\rangle$ is normal in $G$. If $x^{p} \neq 1$, then by Lemma 2.4 $\left\langle x^{p}\right\rangle\left\langle y^{q}\right\rangle \neq\left\langle x^{p}\right\rangle \times\left\langle y^{q}\right\rangle$. Lemma 2.2 implies that $\left\langle y^{q}\right\rangle$ is normal in $\left\langle x^{p}\right\rangle\langle y\rangle$. Thus, $\left\langle x^{p}\right\rangle\left\langle y^{q}\right\rangle=\left\langle x^{p}\right\rangle \times\left\langle y^{q}\right\rangle$, a contradiction. So $x^{p}=1$ and $G$ is of type (I).

(2) Assume that $P$ is cyclic and $Q$ is non-cyclic.

Clearly, if $p<q$, then $Q \unlhd G$, a contradiction. Hence $p>q$.

If $Q$ has two non-cyclic maximal subgroups $Q_{1}$ and $Q_{2}$, then by Lemma 2.2 $P Q_{1}=P \times Q_{1}$ and $P Q_{2}=P \times Q_{2}$. Hence $Q=Q_{1} Q_{2}$ is normal in $G$, a contradiction. Therefore, every maximal subgroup of $Q$ is cyclic or $Q$ has a unique non-cyclic maximal subgroup. Thus, $Q$ is the elementary abelian group of order $q^{2}$, the quaternion group $Q_{8}$ or one of the types of Lemma 2.5

Case 1. Assume $P=\langle z\rangle, Q=\left\langle a^{q}=b^{q}=1,[a, b]=1\right\rangle$. If $z^{p} \neq 1$, then $\left\langle z^{p}\right\rangle Q=\left\langle z^{p}\right\rangle \times Q$ by Lemma 2.2. If the actions of $a$ and $b$ on $P$ by conjugation are both trivial, then $G$ is nilpotent, a contradiction. Therefore, we may assume that the action of $a$ on $P$ by conjugation is non-trivial. By applying Lemma 2.4 $\left\langle z^{p}\right\rangle\langle a\rangle \neq\left\langle z^{p}\right\rangle \times\langle a\rangle$, a contradiction. Hence $z^{p}=1$. If $C_{G}(P)=P$, then $G / C_{G}(P)$ is an elementary abelian group of order $q^{2}$. However, $G / C_{G}(P) \lesssim \operatorname{Aut}(P)$, and $\operatorname{Aut}(P)$ is cyclic, a contradiction. Hence either $a$ or $b$ is contained in $C_{G}(P)$. Let $C_{G}(P)=\langle x\rangle, y^{-1} x y=x^{r}$ with $1<r<p$, where $x=z b$ is a generator of 
$C_{G}(P),|x|=p q, y=a$. Then we have that $p \equiv 1(\bmod q), r \equiv 1(\bmod q)$ and $r^{q} \equiv 1(\bmod p)$. So $G$ is of type (II).

Case 2. Assume $Q=Q_{8}=\left\langle a, b \mid a^{4}=1, b^{2}=a^{2}, b^{-1} a b=a^{-1}\right\rangle$ and $P=\langle z\rangle$. If $z^{p} \neq 1$, then $\left\langle z^{p}\right\rangle Q=\left\langle z^{p}\right\rangle \times Q$ by Lemma 2.2 Using the same argument as in Case 1, we obtain the same contradiction. Hence $z^{p}=1$. Since $P\langle a\rangle$ is an $\mathrm{MSN}^{*}$ group, $\left\langle a^{2}\right\rangle \leqslant C_{G}(P)$ by Lemma 2.2. If $C_{G}(P)=P \times\left\langle a^{2}\right\rangle$, then $G / C_{G}(P)$ is an elementary abelian group of order 4 . Again, there is a contradiction as in Case 1. So $C_{G}(P)$ has an element of order 4 and $C_{G}(P)=\langle x\rangle$ is a cyclic group of order $4 p$. Surely, $G$ has an element $y$ of order 4 such that $y \neq x^{p}$. Now we let $y^{-1} x y=x^{r}$ where $r \not \equiv 1(\bmod 4 p)$. Since $\left(y^{2}\right)^{-1} x y^{2}=x^{r^{2}}=x$, we have $r^{2} \equiv 1(\bmod 4 p)$. By computations, $G=\left\langle x, y \mid x^{4 p}=1, y^{2}=x^{2 p}, y^{-1} x y=x^{-1}\right\rangle$. So $G$ is of type (III).

Case 3. Assume that $P=\langle x\rangle$ and $Q$ is of type (I) with $|Q|=q^{n}$ in Lemma 2.5 Namely, $Q=\left\langle y, z \mid y^{q^{n-1}}=z^{q}=1,[y, z]=1\right\rangle$, where $n \geqslant 3$. Then $Q$ has maximal subgroups $H=\langle y\rangle, K_{0}=\left\langle y^{q}, z\right\rangle$ and $K_{s}=\left\langle y^{q}, z y^{s}\right\rangle=\left\langle z y^{s}\right\rangle$ with $s=$ $1, \ldots, q-1$, where $K_{0}$ is the unique non-cyclic maximal subgroup of $Q$. Lemma 2.2 implies $P K_{0}=P \times K_{0}$. By the hypothesis, $P H \neq P \times H$ and $y$ induces a power automorphism of order $q$ on $P$. Surely, $z \in Z(G)$. Further, by similar arguments as in Case 1 , we have $x^{p}=1$. Hence $G=\langle x, y, z| x^{p}=y^{q^{n-1}}=z^{q}=1, y^{-1} x y=$ $\left.x^{r},[x, z]=1,[y, z]=1\right\rangle$, where $p \equiv 1(\bmod q), r \not \equiv 1(\bmod p), r^{q} \equiv 1(\bmod p)$ with $1<r<p$. So $G$ is of type (IV).

Case 4. Assume that $P=\langle x\rangle$ and $Q$ is of type (II) with $|Q|=q^{n}$ in Lemma 2.5 Namely, $Q=\left\langle y, z \mid y^{q^{n-1}}=z^{q}=1, z^{-1} y z=y^{1+p^{n-2}}\right\rangle$, where $n \geqslant 3$ and $n \geqslant 4$ if $p=2$. Similarly as above, $y$ induces a power automorphism of order $q$ on $P$ and $\langle z\rangle \leqslant C_{G}(P)$. Further, we can prove that $x^{p}=1, y^{-1} x y=x^{r}$, where $p \equiv 1(\bmod q), r \not \equiv 1(\bmod p), r^{q} \equiv 1(\bmod p)$ with $1<r<p$. So $G$ is of type $(\mathrm{V})$.

(3) Assume that $P$ is non-cyclic and $Q=\langle y\rangle$ is a cyclic subgroup of $G$ with $|y|=q^{n}$.

If $G$ is supersolvable, we can assume that $1 \unlhd \cdots \unlhd R \unlhd P \unlhd \cdots \unlhd G$ is a chief series of $G$. By Maschke's theorem [9, 8.1.2], there exists a subgroup $H$ of $P$ such that $P / \Phi(P)=R / \Phi(P) \times H / \Phi(P)$, where $|H / \Phi(P)|=p$ and $H / \Phi(P) \unlhd G / \Phi(P)$. Clearly, $H \unlhd G, H \not \leq R$ and $1 \unlhd H \unlhd P \unlhd G$ is a normal series of $G$. By applying Schreier's refinement theorem [9, 3.1.2], there exists a maximal subgroup $K$ of $P$ such that $K$ is normal in $G$ and $K \neq R$. By the minimality of $G, R Q$ and $K Q$ are both $\mathrm{MSN}^{*}$-groups of $G$. Lemma 2.2 implies that $\left\langle y^{q}\right\rangle$ is normal in $G$, so $G$ is an $\mathrm{MSN}^{*}$-group, a contradiction. Therefore, $G$ is minimal non-supersolvable.

Case 1. If $G$ is also a minimal non-nilpotent group and $P$ is abelian, by applying [2, Theorem 3], $G$ is of one of the types (VI)-(VII).

Case 2. If $G$ is not a minimal non-nilpotent group and $P$ is abelian, by applying [1, Theorems 9, 10], we assume that $G=P Q$, where $P=\left\langle a_{0}, a_{1}, \ldots, a_{q-1}\right\rangle$ is an elementary abelian $p$-group of order $p^{q}, Q=\langle y\rangle$ is cyclic of order $q^{n}, q^{f}$ is the highest power of $q$ dividing $p-1$ and $n>f \geqslant 1$. Define $a_{j}^{y}=a_{j+1}$ for $0 \leqslant j<q-1$ and $a_{q-1}^{y}=a_{0}^{i}$, where $i$ is a primitive $q^{f}$-th root of unity modulo $p$. 
Considering a maximal group $P\left\langle y^{q}\right\rangle$ of $G$, by Lemma 2.2. $\left\langle y^{q^{2}}\right\rangle \leqslant C_{G}(P)$. Hence, $a_{0}^{i^{q}}=a_{0}^{y^{q^{2}}}=a_{0}$. Thus $i^{q} \equiv 1(\bmod p)$, i.e., $f=1, G$ is of type (VIII).

Case 3. If $G$ is not a minimal non-nilpotent group and $P$ is non-abelian, by applying [1, Theorems 9, 10], we may assume that $G=P Q$ such that $P=\left\langle a_{0}, a_{1}\right\rangle$ is an extraspecial group of order $p^{3}$ with exponent $p, Q=\langle y\rangle$ is a cyclic group of order $2^{n}$ with $2^{f}$ the largest power of 2 dividing $p-1$ and $n>f \geqslant 1$, and $a_{0}^{y}=a_{1}$ and $a_{1}^{y}=a_{0}^{i} x$, where $x \in\left\langle\left[a_{0}, a_{1}\right]\right\rangle$ and $i$ is a primitive $2^{f}$-th root of unity modulo $p$.

Since $P\left\langle y^{2}\right\rangle$ and $\Phi(P)\langle y\rangle$ are MSN*-groups, we have that $\left\langle y^{4}\right\rangle \leqslant C_{G}(P)$ and $\left\langle y^{2}\right\rangle \leqslant C_{G}(\Phi(P))$ by Lemma 2.2 . However, $a_{0}^{y^{4}}=a_{0}^{i^{2}} x^{i+1} \neq a_{0}$, a contradiction. Therefore, $G$ is not of the type as above.

(4) Assume that $P$ and $Q$ are both non-cyclic.

Using similar arguments as in Situation (3), we easily have that $G$ is minimal non-supersolvable. By the same arguments as in Situation (2), $Q$ is the elementary abelian group of order $q^{2}$, the quaternion group $Q_{8}$ or one of the two types of Lemma 2.5

Case 1. Let $Q$ be an elementary abelian group of order $q^{2}$. By applying [1, Theorems 9, 10], none of them satisfies a minimal non-MSN*-group.

Case 2. Let $Q=Q_{8}=\left\langle a, b \mid a^{4}=1, b^{2}=a^{2}, b^{-1} a b=a^{-1}\right\rangle$. For maximal subgroups $P\langle a\rangle$ and $P\langle b\rangle$ of $G$, we have $\Phi(Q)=\left\langle a^{2}\right\rangle \leqslant C_{G}(P)$ by Lemma 2.2 By examining Type 6 and Type 7 in [1, Theorems 9, 10], none of them satisfies a minimal non-MSN*-group.

Case 3. Let $Q$ be as in Lemma 2.5 (I) with $|Q|=q^{n}$. Namely, $Q=\langle a, b|$ $\left.a^{q^{n-1}}=b^{q}=1,[a, b]=1\right\rangle$ where $n \geqslant 3$. By similar arguments as Case 2 in Situation (3), none of the types 6-10 in [1, Theorems 9, 10] satisfies a minimal non-MSN*-group.

Case 4. Let $Q$ be as in Lemma 2.5 (II) with $|Q|=q^{n}$. Namely, $Q=\langle a, b|$ $\left.a^{q^{n-1}}=b^{q}=1, b^{-1} a b=a^{1+q^{n-2}}\right\rangle$, where $n \geqslant 3$ and $n \geqslant 4$ if $q=2$. It is clear that $\Phi(Q)=\left\langle a^{q}\right\rangle \leqslant C_{G}(P)$. By examining types 6-10 in [1, Theorems 9, 10], none of them satisfies a minimal non-MSN*-group.

We next consider the case of $G$ with $|\pi(G)|=3$.

Lemma 2.3 implies that $G$ is minimal non-supersolvable. By types 11-12 in [1, Theorems 9, 10], we may assume that $G=P Q R$ with $P \unlhd G, Q$ is neither cyclic nor normal in $G$, and $R \not G$, where $P \in \operatorname{Syl}_{p}(G), Q \in \operatorname{Syl}_{q}(G)$ and $R \in \operatorname{Syl}_{r}(G)$. However, by Lemma 2.2, $Q$ is normal in both $P Q$ and $Q R$, and so $Q$ is normal in $G=\langle P, Q, R\rangle$, a contradiction. Hence none of them satisfies a minimal non-MSN*group.

Conversely, it is clear that a group satisfying one of the types (I)-(VIII) is a minimal non-MSN*-group.

Corollary 3.2. The following statements for a group $G$ are equivalent:

(1) $G$ is a minimal non-MSN*-group.

(2) $G$ is a minimal non-CMSN-group.

(3) $G$ is exactly of one type of Theorem 3.1 
Proof. From Theorem 3.1, it suffices to prove that a minimal non-CMSN-group is a minimal non-MSN*-group.

Let $G$ be a minimal non-CMSN-group. For each maximal subgroup $M$ of $G$, all subgroups of $M$ are CLT-groups by the minimality of $G$. By Lemma 2.6, $M$ is supersolvable, so $G$ is a minimal non-MSN*-group.

\section{ACKNOWLEDGEMENTS}

The authors are very grateful to the referees for their careful reading and giving valuable comments which led to the improvement of this paper.

\section{REFERENCES}

[1] A. Ballester-Bolinches and R. Esteban-Romero, On minimal non-supersoluble groups, Rev. Mat. Iberoam. 23 (2007), no. 1, 127-142. MR 2351128

[2] A. Ballester-Bolinches, R. Esteban-Romero and D.J.S. Robinson, On finite minimal nonnilpotent groups, Proc. Amer. Math. Soc. 133 (2005), no. 12, 3455-3462. MR 2163579.

[3] K. Doerk, Minimal nicht überauflösbare, endliche Gruppen, Math. Z. 91 (1966), 198-205. MR 0191962

[4] P.F. Guo, R.F. Ge, C.G. Shao and X.H. Zhang, A classification of minimal non-MNP-groups, Comm. Algebra 41 (2013), no. 5, 1601-1607. MR 3062812

[5] P.F. Guo and X.Y. Guo, On minimal non-MSN-groups, Front. Math. China 6 (2011), no. 5, 847-854. MR 2836852

[6] J.J. Liu, S.R. Li and J. He, CLT-groups with normal or abnormal subgroups, J. Algebra 362 (2012), 99-106. MR 2921632

[7] D.J. McCarthy, A survey of partial converses to Lagrange's theorem on finite groups, Trans. New York Acad. Sci. 33 (1971), 586-594. MR 0311766

[8] D.H. McLain, The existence of subgroups of given order in finite groups, Math. Proc. Cambridge Philos. Soc. 53 (1957), no. 2, 278-285. MR 0085260

[9] D.J.S. Robinson, A Course in the Theory of Groups, Graduate Texts in Mathematics, 80. Springer-Verlag, New York-Berlin, 1982. MR 0648604.

[10] O.J. Schmidt, Über Gruppen, deren sämtliche Teiler spezielle Gruppen sind, Mat. Sbornik 31 (1924), 366-372.

[11] H.G. Shi and Z.J. Han, On minimal non- $\mathscr{B}$-groups, Rev. Un. Mat. Argentina 55 (2014), no. 2, 101-106. MR 3285343

[12] S. Srinivasan, Two sufficient conditions for supersolvability of finite groups, Israel J. Math. 35 (1980), no. 3, 210-214. MR 0576471

[13] G.L. Walls, Groups with maximal subgroups of Sylow subgroups normal, Israel J. Math. 43 (1982), no. 2, 166-168. MR 0689976

[14] M. Weinstein (Ed.), Between Nilpotent and Solvable, Polygonal Publishing House, Passaic, NJ, 1982. MR 0655785

[15] Q.H. Zhang, L.F. Wang and P.F. Guo, On the structure of some finite groups, Southeast Asian Bull. Math. 30 (2006), no. 5, 995-1002. MR 2287022 
Pengfei Guo

College of Mathematics and Statistics, Hainan Normal University, Haikou, Hainan 571158,

P. R. China

guopf999@163.com

Xingqiang Xiu

College of Mathematics and Statistics, Hainan Normal University, Haikou, Hainan 571158,

P. R. China

xiu_hainu@163.com

Guangjun $X u^{凶}$

School of Mathematics, Zunyi Normal University, Zunyi, Guizhou 563006, P. R. China

gjxu_shu@hotmail.com

Received: June 6, 2018

Accepted: December 18, 2018 